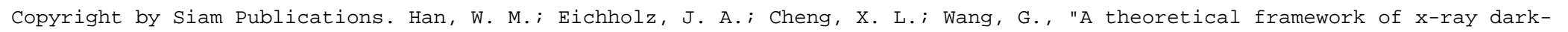
field tomography," SIAM J. Appl. Math., 71(5), 1557-1577, (2011). DOI: 10.1137/100809039

\title{
A THEORETICAL FRAMEWORK OF X-RAY DARK-FIELD TOMOGRAPHY*
}

\author{
WEIMIN $\mathrm{HAN}^{\dagger}$, JOSEPH A. EICHHOLZ ${ }^{\dagger}$, XIAOLIANG CHENG ${ }^{\ddagger}$, AND GE WANG§
}

\begin{abstract}
X-ray mammography is currently the most prevalent imaging modality for screening and diagnosis of breast cancers. However, its success is limited by the poor differentiation between healthy and diseased tissues in the mammogram. A potentially prominent imaging modality is based on the significant difference of x-ray scattering behaviors between tumor and normal tissues. Driven by major practical needs for better x-ray imaging, exploration into contrast mechanisms other than attenuation has been active for decades, e.g., in terms of scattering, which is also known as dark-field tomography. This paper provides a preliminary theoretical study of x-ray dark-field tomography (XDT) assuming the spectral x-ray detection technology. For XDT, the modified Leakeas-Larsen equation (MLLE) is an appropriate approximation of the radiative transfer equation (RTE) for a highly forward-peaked medium with small but sufficient amounts of large-angle scattering. Properties of the MLLE are studied, such as existence of a unique solution and positivity of the solution. MLLE and its discrete analogues can be solved naturally with an iteration procedure, and convergence of the iteration procedure is shown. XDT, as an inverse parameter problem with MLLE as the forward model, is then studied. Numerical discretization schemes of MLLE and the associated XDT are introduced. Simulation results are reported on several numerical examples for MLLE and for XDT. The paper concludes with some remarks on research topics for further study of XDT.
\end{abstract}

Key words. x-ray dark-field tomography, modified Leakeas-Larsen equation, existence, uniqueness, stability, iteration, numerical method

AMS subject classifications. 35R30, 35M10, 65N21

DOI. $10.1137 / 100809039$

1. Introduction. X-ray mammography is currently the most prevalent imaging modality for screening and diagnosis of breast cancers. The use of mammography results in a $25-30 \%$ decreased mortality rate in screened women [25]. However, a multi-institutional trial funded by the American College of Radiology Imaging Network (ACRIN) suggested that about $30 \%$ of cancers were not detected by screening mammography (false negatives), and $70-90 \%$ of biopsies performed based on suspicious mammograms were negative (false positives) [29]. The key factor that limits mammography success rate is the poor differentiation between healthy and diseased tissues in the mammogram. Although x-ray computed tomography (CT) of the breast can potentially improve diagnostic accuracy over mammography $[14,15]$, the stateof-the-art breast CT scanner is still based on the attenuation mechanism. As a result, the use of breast $\mathrm{CT}$ requires an intravenous contrast medium and a high radiation

*Received by the editors September 20, 2010; accepted for publication (in revised form) May 31, 2011; published electronically September 6, 2011. The work of the first and fourth authors was partially supported by NIH/NHLBI-HL098912.

http://www.siam.org/journals/siap/71-5/80903.html

$\dagger$ Department of Mathematics \& Program in Applied Mathematical and Computational Sciences, University of Iowa, Iowa City, IA 52242 (weimin-han@uiowa.edu, joseph-eichholz@uiowa.edu). The work of the first and second authors was partially supported by NSF DMS-0602242.

$\ddagger$ Department of Mathematics, Zhejiang University, Hangzhou 310027, China (xiaoliangcheng@ zju.edu.cn). The work of this author was partially supported by the National Science Foundation of China, grant 10871179 .

${ }^{\S}$ Division of Biomedical Imaging, Virginia Tech-Wake Forest University School of Biomedical Engineering and Sciences, Virginia Polytechnic Institute and State University, Blacksburg, VA 24061 (ge-wang@ieee.org). The work of this author was partially supported by NIH/NIBIB-EB011785.

1557 
dose, since elemental composition is almost uniform with little density variation in breast tissues. Still, it is rather difficult for breast CT to discern early-stage breast cancers.

The main components of the breast are (1) the adipose tissue consisting of large fatty cells and (2) the connective tissue containing fibrous collagen. Fibril ordering is basically absent within tumors. Tissue remodeling is a crucial step during a malignant transformation of epithelial cells; carcinoma invasion is accompanied by destruction and synthesis of fibrillary and nonfibrillary matrix proteins, creating characteristic desmoplastic stromal changes. The dense collagenous tumor core is firm in consistency, whereas the invasive tumor front is rich in looser fibrils. Invasive carcinoma cells migrate along collagen strands. These are attributed to structural degradation of invaded collagen or a low degree of ordering of the newly formed collagen among the adipocytes in the fatty tissue, where collagen is accompanied by invading cancer cells. Hence, a significant structural variation occurs with respect to supramolecular collagen architecture in tumors, resulting in a significant difference of x-ray scattering behaviors between tumor and normal tissues [12]. Every tissue component gives rise to a characteristic x-ray scattering (SAXS) pattern. Such scattering signals reveal critical information on ultrafine features of cellular and subcellular structures, and have unique diagnostic values of molecules, cells, and their clusters which are also known as basic functional units of $100-200 \mathrm{~m}$ in diameter [7, 23]. Most importantly, $\mathrm{x}$-ray scattering properties of tumors are significantly different from those of healthy tissue. Hence, x-ray scattering imaging provides a new contrast mechanism and would well complement attenuation-based x-ray imaging [23, 28].

Driven by major practical needs for better x-ray imaging, exploration into contrast mechanisms other than attenuation has been active for decades, especially in terms of scattering and refraction of x-rays, which are also known as dark-field and phase-contrast imaging, respectively [30]. Up to now, x-ray scattering-based imaging has been limited to in vitro studies, incapable of volumetric cone-beam scanning, lacking rigorous reconstruction theory, and making little progress into clinical practice. Since 2006, grating-based x-ray dark-field and phase-contrast tomography has been developed using a hospital-grade x-ray tube, instead of a synchrotron facility or micro-focus tube [28]. This technology utilizes optical interference principles to yield high-quality dark-field images. The boundaries and interfaces in the biological tissues produce strong signals in dark-field images, indicating detailed structural contours. Moreover, dark-field images have greater signal-to-noise ratios in soft tissues than bright-field counterparts acquired with the same incident x-ray dose. However, the major problems with this grating-based approach are small sample size, long imaging time, and high fabrication cost.

Currently, we are studying novel dark-field detection and tomography schemes for screening and diagnosis of breast cancers assuming the spectral x-ray detection technology, as is described in $[2,9]$. In this paper, we provide a preliminary theoretical study of x-ray dark-field tomography (XDT), employing the modified LeakeasLarsen equation (MLLE) [20, 19]. Our proposed theory and biomedical applications can match well, thanks to the emerging x-ray energy-discriminating photon-counting detection technology. The MLLE assumes that the radiative flux strongly favors the forward direction and that there is a small but sufficient amount of large-angle scattering. In the biomedical x-ray energy range, the Compton scattering is more or less isotropic and can be quite strong. When the Compton components are significant and an object to be imaged is relatively large, the assumption behind MLLE is not valid with a photon-integrating detector array. Fortunately, the energy-sensitive de- 
tectors such as the Medipix family of photon-processing detectors [22, 9] can be used to solve this problem. It is well known that Compton scattering is inelastic, and the larger the scattering angle is from the primary beam direction, the more the photon energy will be lost. With a Medipix detector array and a relatively monochromatic source, large-angle interference can be effectively filtered out, if needed, for MLLE to be applicable.

The rest of the paper is structured as follows. In section 2, we review the radiative transfer equation (RTE) and MLLE. In section 3, we study properties of MLLE, such as existence of a unique solution and positivity of the solution. The MLLE can be solved naturally with an iteration procedure, and we show convergence of the iteration procedure in section 4. Although the convergence is shown only at the continuous level, it holds also in applying the iteration method to solve discretized systems of MLLE. In section 5, we study XDT problems, with angularly resolved measurement or angularly averaged measurement. An XDT problem is an inverse problem for determination of the scattering parameter, with the MLLE as the forward model. In section 6, we describe numerical discretization schemes of MLLE and the associated XDT. In section 7 , we present simulation results from several examples. Finally, in section 8 , some remarks on further research are given.

2. The RTE and MLLE. The propagation of light within biological media is described by the RTE $[4,26]$. In this section, we review the RTE and introduce its simplified approximation, MLLE, for strongly forward-peaked media.

Let $X$ be a domain in $\mathbb{R}^{3}$ with a Lipschitz boundary $\partial X$, and let $\Omega$ be the unit sphere in $\mathbb{R}^{3}$. For each fixed direction $\boldsymbol{\omega} \in \Omega$ introduce a new Cartesian coordinate system $\left(z_{1}, z_{2}, s\right)$ by the relations $\boldsymbol{x}=\boldsymbol{z}+s \boldsymbol{\omega}, \boldsymbol{z}=z_{1} \boldsymbol{\omega}_{1}+z_{2} \boldsymbol{\omega}_{2}$, where $\left(\boldsymbol{\omega}, \boldsymbol{\omega}_{1}, \boldsymbol{\omega}_{2}\right)$ is an orthonormal basis of $\mathbb{R}^{3}, z_{1}, z_{2}, s \in \mathbb{R}$. With respect to this new coordinate system, we denote by $X_{\boldsymbol{\omega}}$ the projection of $X$ on the plane $s=0$ in $\mathbb{R}^{3}$, and by $X_{\boldsymbol{\omega}, \boldsymbol{z}}$ $\left(\boldsymbol{z} \in X_{\boldsymbol{\omega}}\right)$ the intersection of the straight line $\{\boldsymbol{z}+s \boldsymbol{\omega} \mid s \in \mathbb{R}\}$ with $X$. We assume that the domain $X$ is such that for any $(\boldsymbol{\omega}, \boldsymbol{z})$ with $\boldsymbol{z} \in X_{\boldsymbol{\omega}}, X_{\boldsymbol{\omega}, \boldsymbol{z}}$ is the union of a finite number of line segments:

$$
X_{\boldsymbol{\omega}, \boldsymbol{z}}=\bigcup_{i=1}^{N(\boldsymbol{\omega}, \boldsymbol{z})}\left\{\boldsymbol{z}+s \boldsymbol{\omega} \mid s \in\left(s_{i,-}, s_{i,+}\right)\right\} .
$$

Here $s_{i, \pm}=s_{i, \pm}(\boldsymbol{\omega}, \boldsymbol{z})$ depend on $\boldsymbol{\omega}$ and $\boldsymbol{z}$, and $\boldsymbol{x}_{i, \pm}:=\boldsymbol{z}+s_{i, \pm} \boldsymbol{\omega}$ are the intersection points of the line $\{\boldsymbol{z}+s \boldsymbol{\omega} \mid s \in \mathbb{R}\}$ with $\partial X$. We further assume $\sup _{\boldsymbol{\omega}, \boldsymbol{z}} N(\boldsymbol{\omega}, \boldsymbol{z})<\infty$, known as a generalized convexity condition. As an example, for a convex domain $X$, $\sup _{\boldsymbol{\omega}, \boldsymbol{z}} N(\boldsymbol{\omega}, \boldsymbol{z})=1$. We then introduce the following subsets of $\partial X$,

$$
\partial X_{\boldsymbol{\omega}, \pm}=\left\{\boldsymbol{z}+s_{i, \pm} \boldsymbol{\omega} \mid 1 \leq i \leq N(\boldsymbol{\omega}, \boldsymbol{z}), \boldsymbol{z} \in X_{\boldsymbol{\omega}}\right\},
$$

and the following incoming and outgoing boundaries as subsets of $\Gamma=\partial X \times \Omega$ :

$$
\Gamma_{-}=\left\{(\boldsymbol{x}, \boldsymbol{\omega}) \mid \boldsymbol{x} \in \partial X_{\boldsymbol{\omega},-}, \boldsymbol{\omega} \in \Omega\right\}, \quad \Gamma_{+}=\left\{(\boldsymbol{x}, \boldsymbol{\omega}) \mid \boldsymbol{x} \in \partial X_{\boldsymbol{\omega},+}, \boldsymbol{\omega} \in \Omega\right\} .
$$

Denote by $d \sigma(\boldsymbol{\omega})$ the infinitesimal area element on the unit sphere $\Omega$. In the spherical coordinate system, $\boldsymbol{\omega}=(\sin \theta \cos \psi, \sin \theta \sin \psi, \cos \theta)^{T}$ for $0 \leq \theta \leq \pi$ and $0 \leq \psi<2 \pi$, we have $d \sigma(\boldsymbol{\omega})=\sin \theta d \theta d \psi$. We need an integral operator $S$ defined by

$$
(S u)(\boldsymbol{x}, \boldsymbol{\omega})=\int_{\Omega} \eta(\boldsymbol{x}, \boldsymbol{\omega} \cdot \hat{\boldsymbol{\omega}}) u(\boldsymbol{x}, \hat{\boldsymbol{\omega}}) d \sigma(\hat{\boldsymbol{\omega}})
$$


with $\eta$ a nonnegative normalized phase function:

$$
\int_{\Omega} \eta(\boldsymbol{x}, \boldsymbol{\omega} \cdot \hat{\boldsymbol{\omega}}) d \sigma(\hat{\boldsymbol{\omega}})=1 \quad \forall \boldsymbol{x} \in X, \boldsymbol{\omega} \in \Omega .
$$

In many applications, the function $\eta$ is independent of $\boldsymbol{x}$. However, in our general study, we allow $\eta$ to depend on $\boldsymbol{x}$. Moreover, we can allow $\eta$ to be a general function of $\boldsymbol{x}, \boldsymbol{\omega}$, and $\hat{\boldsymbol{\omega}}$, i.e., in the form $\eta(\boldsymbol{x}, \boldsymbol{\omega}, \hat{\boldsymbol{\omega}})$. One well-known example is the HenyeyGreenstein phase function (cf. [18])

$$
\eta(t)=\frac{1-g^{2}}{4 \pi\left(1+g^{2}-2 g t\right)^{3 / 2}}, \quad t \in[-1,1],
$$

where the parameter $g \in(-1,1)$ is the anisotropy factor of the scattering medium. Note that $g=0$ for isotropic scattering, $g>0$ for forward scattering, and $g<0$ for backward scattering.

With the above notation, a boundary value problem of the RTE reads (cf. [1, 21])

$$
\begin{aligned}
\boldsymbol{\omega} \cdot \nabla u+\mu_{t} u & =\mu_{s} S u+f \quad \text { in } X \times \Omega, \\
u & =0 \quad \text { on } \Gamma_{-} .
\end{aligned}
$$

Here $\mu_{t}=\mu_{a}+\mu_{s}, \mu_{a}$ is the macroscopic absorption cross section, $\mu_{s}$ is the macroscopic scattering cross section, and $f$ is a source function. We assume

$$
\begin{aligned}
& \mu_{t}, \mu_{s} \in L^{\infty}(X), \quad \mu_{s} \geq 0 \text { a.e. in } X, \quad \mu_{t}-\mu_{s} \geq c_{0}>0 \text { a.e. in } X, \\
& f(\boldsymbol{x}, \boldsymbol{\omega}) \in L^{2}(X \times \Omega) \text { and is continuous w.r.t. } \boldsymbol{\omega} \in \Omega \text { for a.e. } \boldsymbol{x} \in X .
\end{aligned}
$$

These assumptions are naturally valid in applications; the last part of (2.6) means that the absorption effect is not negligible. The homogeneous boundary condition (2.5) corresponds to a vacuum setting around $X$. It is equally acceptable to consider a general incoming flux boundary condition $u=u_{\text {in }}$ on $\Gamma_{-}$for a given function $u_{\text {in }}$.

It is known [1] that the problem (2.4)-(2.5) has a unique solution $u \in H^{1,2}(X \times \Omega)$, where

$$
H^{1,2}(X \times \Omega):=\left\{v \in L^{2}(X \times \Omega) \mid \boldsymbol{\omega} \cdot \nabla v \in L^{2}(X \times \Omega)\right\},
$$

$\boldsymbol{\omega} \cdot \boldsymbol{\nabla} v$ being the generalized directional derivative of $v$ in the direction $\boldsymbol{\omega}$ (cf. [1]).

Later, we also need function spaces $L^{2}\left(\Gamma_{ \pm}\right)$on $\Gamma_{ \pm}$. They are Hilbert spaces of functions $v$ on $\Gamma_{ \pm}$with inner products

$$
(u, v)_{L^{2}\left(\Gamma_{ \pm}\right)}:=\int_{\Omega} d \sigma(\boldsymbol{\omega}) \int_{X_{\boldsymbol{\omega}}} \sum_{i=1}^{N(\boldsymbol{\omega}, \boldsymbol{z})} u\left(\boldsymbol{z}+s_{i, \pm} \boldsymbol{\omega}, \boldsymbol{\omega}\right) v\left(\boldsymbol{z}+s_{i, \pm} \boldsymbol{\omega}, \boldsymbol{\omega}\right) d z
$$

and corresponding norms $\|v\|_{L^{2}\left(\Gamma_{+}\right)}$. We have the following statement for the trace of an $H^{1,2}(X \times \Omega)$ function [1, Lemma 2.2]. If $v \in H^{1,2}(X \times \Omega)$ and $\left.v\right|_{\Gamma_{-}} \in L^{2}\left(\Gamma_{-}\right)$, then $\left.v\right|_{\Gamma_{+}} \in L^{2}\left(\Gamma_{+}\right)$and, for some constant $c$ depending only on $X$,

$$
\|v\|_{L^{2}\left(\Gamma_{+}\right)} \leq c\left[\|v\|_{H^{1,2}(X \times \Omega)}+\|v\|_{L^{2}\left(\Gamma_{-}\right)}\right] .
$$

The statement remains valid by switching $\Gamma_{+}$and $\Gamma_{-}$.

Inverse problems for the RTE have been studied substantially in recent years; see, e.g., [8] and the references therein. Due to the high dimension and the integrodifferential form of the equation, it is very difficult to solve the RTE accurately, 
especially for highly forward-peaked biological media, as is the case for our applications. Hence, several simplified approximations of the RTE have been introduced. For $\eta$ strongly forward peaked - for the Henyey-Greenstein phase function (2.3), this means $g$ is close to 1 - a simplification can be introduced through Taylor's expansion of $u(\boldsymbol{x}, \hat{\boldsymbol{\omega}})$ for $\hat{\boldsymbol{\omega}}$ about $\boldsymbol{\omega}$ [19]. The result is the Fokker-Planck equation

$$
\boldsymbol{\omega} \cdot \nabla u+\mu_{a} u=\frac{\mu_{t r}}{2} \Delta^{*} u+f \quad \text { in } X \times \Omega,
$$

where $\Delta^{*}$ is the Laplace-Beltrami operator, $\mu_{t r}=\mu_{s}(1-g)$ is the transport cross section, and the degree of anisotropy $g$ is defined by

$$
g(\boldsymbol{x})=2 \pi \int_{-1}^{1} t \eta(\boldsymbol{x}, t) d t .
$$

Note that the integral operator $S$ is bounded, whereas the differential operator on the right-hand side of (2.9) is unbounded. To address this issue, Leakeas and Larsen [20] propose to approximate the RTE by

$$
\boldsymbol{\omega} \cdot \boldsymbol{\nabla} u+\mu_{a} u=\mu_{s} \beta \Delta^{*}\left(I-\alpha \Delta^{*}\right)^{-1} u+f \quad \text { in } X \times \Omega,
$$

where the parameters $\alpha$ and $\beta$ are chosen so that the differential operator $\beta \Delta^{*}(I-$ $\left.\alpha \Delta^{*}\right)^{-1}$ in (2.10) and the integral operator $S$ in (2.4) have similar behavior for some of their eigenvalues. The MLLE adopted in this paper is of the form [19]

$$
\boldsymbol{\omega} \cdot \boldsymbol{\nabla} u+\mu_{t} u=\mu_{s}\left(I-\alpha \Delta^{*}\right)^{-1} u+f \quad \text { in } X \times \Omega .
$$

Here, $\alpha(\boldsymbol{x})$ is a positively valued function of $\boldsymbol{x}$. For the Henyey-Greenstein phase function (2.3), $\alpha=(1-g) /(2 g)$ is a constant. Equation (2.11) is supplemented by the following boundary condition:

$$
u=u_{\text {in }} \quad \text { on } \Gamma_{-} .
$$

We assume

$$
u_{\text {in }} \in L^{2}\left(\Gamma_{-}\right) .
$$

Equation (2.11) can be equivalently written as a system

$$
\begin{aligned}
w & =\left(I-\alpha \Delta^{*}\right)^{-1} u \quad \text { in } X \times \Omega, \\
\boldsymbol{\omega} \cdot \boldsymbol{\nabla} u+\mu_{t} u & =\mu_{s} w+f \quad \text { in } X \times \Omega .
\end{aligned}
$$

3. Well-posedness of the MLLE. In this section, we show that, under the assumptions (2.6)-(2.7), the MLLE problem (2.11)-(2.12) has a unique solution and that the solution is stable with respect to the source function and the boundary condition. At the end of the section, we prove a positivity property which is required for the model (2.11)-(2.12) to be physically meaningful.

Without loss of generality, we prove the well-posedness result for the case where $X$ is a convex domain in $\mathbb{R}^{3}$. This allows us to simplify various expressions and to focus on the essentials in the argument. All the discussions in the rest of the paper can be extended to the general case where the domain $X$ satisfies the generalized convexity condition. Thus for each $\boldsymbol{\omega} \in \Omega$ and $\boldsymbol{z} \in X_{\boldsymbol{\omega}}, X_{\boldsymbol{\omega}, \boldsymbol{z}}:=\left\{\boldsymbol{z}+s \boldsymbol{\omega} \mid s \in\left(s_{-}, s_{+}\right)\right\}$is a line segment, where $s_{ \pm}=s_{ \pm}(\boldsymbol{\omega}, \boldsymbol{z})$ depend on $\boldsymbol{\omega}$ and $\boldsymbol{z}$, and $\boldsymbol{x}_{ \pm}:=\boldsymbol{z}+s_{ \pm} \boldsymbol{\omega}$ are the intersection points of the line $\{\boldsymbol{z}+s \boldsymbol{\omega} \mid s \in \mathbb{R}\}$ with $\partial X$. 
First, we convert the MLLE problem (2.11)-(2.12) to a fixed-point formulation. In the following, we write $s_{ \pm}$instead of $s_{ \pm}(\boldsymbol{\omega}, \boldsymbol{z})$ wherever there is no danger of confusion. We write (2.15) as

$(3.1)$

$\frac{\partial}{\partial s} u(\boldsymbol{z}+s \boldsymbol{\omega}, \boldsymbol{\omega})+\mu_{t}(\boldsymbol{z}+s \boldsymbol{\omega}) u(\boldsymbol{z}+s \boldsymbol{\omega}, \boldsymbol{\omega})=\mu_{s}(\boldsymbol{z}+s \boldsymbol{\omega}) w(\boldsymbol{z}+s \boldsymbol{\omega}, \boldsymbol{\omega})+f(\boldsymbol{z}+s \boldsymbol{\omega}, \boldsymbol{\omega})$ and multiply it by an integrating factor $\lambda(s):=\exp \left(\int_{s_{-}}^{s} \mu_{t}(\boldsymbol{z}+s \boldsymbol{\omega}) d s\right)$ to obtain

$$
\frac{\partial}{\partial s}[\lambda(s) u(\boldsymbol{z}+s \boldsymbol{\omega}, \boldsymbol{\omega})]=\lambda(s)\left[\mu_{s}(\boldsymbol{z}+s \boldsymbol{\omega}) w(\boldsymbol{z}+s \boldsymbol{\omega}, \boldsymbol{\omega})+f(\boldsymbol{z}+s \boldsymbol{\omega}, \boldsymbol{\omega})\right] .
$$

Integrate this equation from $s_{-}$to $s$ :

$$
\begin{aligned}
\lambda(s) u(\boldsymbol{z}+s \boldsymbol{\omega}, \boldsymbol{\omega})-u_{\text {in }}(\boldsymbol{z} & \left.+s_{-} \boldsymbol{\omega}, \boldsymbol{\omega}\right) \\
& =\int_{s_{-}}^{s} \lambda(t)\left[\mu_{s}(\boldsymbol{z}+t \boldsymbol{\omega}) w(\boldsymbol{z}+t \boldsymbol{\omega}, \boldsymbol{\omega})+f(\boldsymbol{z}+t \boldsymbol{\omega}, \boldsymbol{\omega})\right] d t .
\end{aligned}
$$

Thus,

$$
u=A u+F
$$

where

$$
\begin{aligned}
A u(\boldsymbol{z}+s \boldsymbol{\omega}, \boldsymbol{\omega}) & =\lambda(s)^{-1} \int_{s_{-}}^{s} \lambda(t) \mu_{s}(\boldsymbol{z}+t \boldsymbol{\omega}) w(\boldsymbol{z}+t \boldsymbol{\omega}, \boldsymbol{\omega}) d t \\
F(\boldsymbol{z}+s \boldsymbol{\omega}, \boldsymbol{\omega}) & =\lambda(s)^{-1}\left[u_{\mathrm{in}}\left(\boldsymbol{z}+s_{-} \boldsymbol{\omega}, \boldsymbol{\omega}\right)+\int_{s_{-}}^{s} \lambda(t) f(\boldsymbol{z}+t \boldsymbol{\omega}, \boldsymbol{\omega}) d t\right] .
\end{aligned}
$$

Reversing the above procedure, we can derive (3.1) from (3.2).

We will show that the operator $A$ is a contraction under suitable norms. We start with

$$
\begin{aligned}
\int_{s_{-}}^{s_{+}} \mu_{t}(\boldsymbol{z}+s \boldsymbol{\omega})|A u(\boldsymbol{z}+s \boldsymbol{\omega}, \boldsymbol{\omega})|^{2} d s \leq & \int_{s_{-}}^{s_{+}} \frac{\mu_{t}(\boldsymbol{z}+s \boldsymbol{\omega})}{\lambda(s)^{2}}\left[\int_{s_{-}}^{s} \lambda(t) \mu_{s}(\boldsymbol{z}+t \boldsymbol{\omega}) d t\right] \\
& \cdot\left[\int_{s_{-}}^{s} \lambda(t) \mu_{s}(\boldsymbol{z}+t \boldsymbol{\omega})|w(\boldsymbol{z}+t \boldsymbol{\omega}, \boldsymbol{\omega})|^{2} d t\right] d s .
\end{aligned}
$$

Define

$$
\kappa:=\sup _{\boldsymbol{x} \in X} \frac{\mu_{s}(\boldsymbol{x})}{\mu_{t}(\boldsymbol{x})} .
$$

The assumption (2.6) implies $\kappa<1$. Since

$$
\int_{s_{-}}^{s} \lambda(t) \mu_{s}(\boldsymbol{z}+t \boldsymbol{\omega}) d t \leq \kappa \int_{s_{-}}^{s} \lambda(t) \mu_{t}(\boldsymbol{z}+t \boldsymbol{\omega}) d t=\kappa(\lambda(s)-1)<\kappa \lambda(s),
$$

we have

$$
\begin{aligned}
& \int_{s_{-}}^{s_{+}} \mu_{t}(\boldsymbol{z}+s \boldsymbol{\omega})|A u(\boldsymbol{z}+s \boldsymbol{\omega}, \boldsymbol{\omega})|^{2} d s \\
& \quad \leq \kappa \int_{s_{-}}^{s_{+}} \lambda(s)^{-1} \mu_{t}(\boldsymbol{z}+s \boldsymbol{\omega}) \int_{s_{-}}^{s} \lambda(t) \mu_{s}(\boldsymbol{z}+t \boldsymbol{\omega})|w(\boldsymbol{z}+t \boldsymbol{\omega}, \boldsymbol{\omega})|^{2} d t d s \\
& \quad=\kappa \int_{s_{-}}^{s_{+}} \lambda(t) \mu_{s}(\boldsymbol{z}+t \boldsymbol{\omega})|w(\boldsymbol{z}+t \boldsymbol{\omega}, \boldsymbol{\omega})|^{2}\left[\int_{t}^{s_{+}} \lambda(s)^{-1} \mu_{t}(\boldsymbol{z}+s \boldsymbol{\omega}) d s\right] d t
\end{aligned}
$$

Copyright (c) by SIAM. Unauthorized reproduction of this article is prohibited. 
Now

$$
\int_{t}^{s_{+}} \lambda(s)^{-1} \mu_{t}(\boldsymbol{z}+s \boldsymbol{\omega}) d s=\lambda(t)^{-1}-\lambda\left(s_{+}\right)^{-1}<\lambda(t)^{-1}
$$

and we obtain

$$
\begin{aligned}
\int_{s_{-}}^{s_{+}} \mu_{t}(\boldsymbol{z}+s \boldsymbol{\omega})|A u(\boldsymbol{z}+s \boldsymbol{\omega}, \boldsymbol{\omega})|^{2} d s & \leq \kappa \int_{s_{-}}^{s_{+}} \mu_{s}(\boldsymbol{z}+t \boldsymbol{\omega})|w(\boldsymbol{z}+t \boldsymbol{\omega}, \boldsymbol{\omega})|^{2} d t \\
& \leq \kappa^{2} \int_{s_{-}}^{s_{+}} \mu_{t}(\boldsymbol{z}+t \boldsymbol{\omega})|w(\boldsymbol{z}+t \boldsymbol{\omega}, \boldsymbol{\omega})|^{2} d t
\end{aligned}
$$

Integrating the above inequality first with respect to $z \in X_{\boldsymbol{\omega}}$ and then with respect to $\boldsymbol{\omega} \in \Omega$, we have thus proved the inequality

$$
\left\|\mu_{t}^{1 / 2} A u\right\|_{L^{2}(X \times \Omega)} \leq \kappa\left\|\mu_{t}^{1 / 2} w\right\|_{L^{2}(X \times \Omega)} .
$$

Returning to the definition (2.14), we have, equivalently,

$$
\left(I-\alpha \Delta^{*}\right) w=u \quad \text { in } X \times \Omega .
$$

For almost every $\boldsymbol{x} \in X$,

$$
w(\boldsymbol{x}, \cdot) \in H^{1}(\Omega), \quad \int_{\Omega}\left(w v+\alpha \nabla^{*} w \cdot \nabla^{*} v\right) d \sigma(\boldsymbol{\omega})=\int_{\Omega} u v d \sigma(\boldsymbol{\omega}) \quad \forall v \in H^{1}(\Omega) .
$$

Here $H^{1}(\Omega):=\left\{v \in L^{2}(\Omega)|| \nabla^{*} v \mid \in L^{2}(\Omega)\right\}$ and $\boldsymbol{\nabla}^{*}$ is the Beltrami gradient operator on $\Omega$. For a function $v$ defined on $\Omega, v^{*}(\boldsymbol{x}):=v(\boldsymbol{x} /|\boldsymbol{x}|)$ is defined on $\Omega_{1}:=\left\{\boldsymbol{x} \in \mathbb{R}^{3}|| \boldsymbol{x} \mid \in[1 / 2,3 / 2]\right\}$, and we have the relation [24]

$$
\left.\boldsymbol{\nabla} v^{*}(r \boldsymbol{\omega})\right|_{r=1}=\nabla^{*} v(\boldsymbol{\omega}), \quad \boldsymbol{\omega} \in \Omega .
$$

For a function $v \in H^{1}\left(\Omega_{1}\right)$, we have $v^{-}:=\min (v, 0) \in H^{1}\left(\Omega_{1}\right)$ [13, Lemma 7.6]. We then deduce, with the help of (3.6), that $v \in H^{1}(\Omega)$ implies $v^{-} \in H^{1}(\Omega)$. This result is used in the proof of Proposition 3.2 below.

Since $\alpha=\alpha(\boldsymbol{x})>0$, for any given $u(\boldsymbol{x}, \cdot) \in L^{2}(\Omega)$, the problem (3.5) has a unique solution $w(\boldsymbol{x}, \cdot) \in H^{1}(\Omega)$ by the Lax-Milgram lemma (cf. [5, p. 336], [10]). Taking $v(\boldsymbol{\omega})=w(\boldsymbol{x}, \boldsymbol{\omega})$ in $(3.5)$,

$$
\int_{\Omega}\left(|w|^{2}+\alpha\left|\nabla^{*} w\right|^{2}\right) d \sigma(\boldsymbol{\omega})=\int_{\Omega} u w d \sigma(\boldsymbol{\omega})
$$

Thus,

$$
\int_{\Omega}\left(|w|^{2}+2 \alpha\left|\nabla^{*} w\right|^{2}\right) d \sigma(\boldsymbol{\omega}) \leq \int_{\Omega}|u|^{2} d \sigma(\boldsymbol{\omega}) .
$$

In particular,

$$
\int_{\Omega}|w|^{2} d \sigma(\boldsymbol{\omega}) \leq \int_{\Omega}|u|^{2} d \sigma(\boldsymbol{\omega})
$$

Therefore,

$$
\left\|\mu_{t}^{1 / 2} w\right\|_{L^{2}(X \times \Omega)} \leq\left\|\mu_{t}^{1 / 2} u\right\|_{L^{2}(X \times \Omega)} .
$$

Copyright (c) by SIAM. Unauthorized reproduction of this article is prohibited. 
Combining (3.4) and (3.8), we see that the operator $A: L^{2}(X \times \Omega) \rightarrow L^{2}(X \times \Omega)$ is contractive with respect to the weighted norm $\left\|\mu_{t}^{1 / 2} \cdot\right\|_{L^{2}(X \times \Omega)}$ :

$$
\left\|\mu_{t}^{1 / 2} A u\right\|_{L^{2}(X \times \Omega)} \leq \kappa\left\|\mu_{t}^{1 / 2} u\right\|_{L^{2}(X \times \Omega)} .
$$

Note that from the assumptions on $\mu_{t}$, the weighted norm $\left\|\mu_{t}^{1 / 2} \cdot\right\|_{L^{2}(X \times \Omega)}$ is equivalent to the regular norm $\|\cdot\|_{L^{2}(X \times \Omega)}$. By an application of the Banach fixed-point theorem (cf. [5, p. 209] or [31]), we conclude that (3.2) has a unique solution $u \in L^{2}(X \times \Omega)$. We further deduce from (2.15) that $\boldsymbol{\omega} \cdot \boldsymbol{\nabla} u(\boldsymbol{x}, \boldsymbol{\omega}) \in L^{2}(X \times \Omega)$. Therefore, the solution $u \in H^{1,2}(X \times \Omega)$. Using (3.9),

$$
\begin{aligned}
\left\|\mu_{t}^{1 / 2} u\right\|_{L^{2}(X \times \Omega)} & \leq\left\|\mu_{t}^{1 / 2} A u\right\|_{L^{2}(X \times \Omega)}+\left\|\mu_{t}^{1 / 2} F\right\|_{L^{2}(X \times \Omega)} \\
& \leq \kappa\left\|\mu_{t}^{1 / 2} u\right\|_{L^{2}(X \times \Omega)}+c\left[\left\|u_{\mathrm{in}}\right\|_{L^{2}\left(\Gamma_{-}\right)}+\|f\|_{L^{2}(X \times \Omega)}\right] .
\end{aligned}
$$

So we have the bound

$$
\|u\|_{L^{2}(X \times \Omega)} \leq c\left[\left\|u_{\mathrm{in}}\right\|_{L^{2}\left(\Gamma_{-}\right)}+\|f\|_{L^{2}(X \times \Omega)}\right] .
$$

Using (2.15), we obtain

$$
\boldsymbol{\omega} \cdot \boldsymbol{\nabla} u=\mu_{s} w-\mu_{t} u+f
$$

Then we can improve the bound (3.10) to the following:

$$
\|u\|_{H^{1,2}(X \times \Omega)} \leq c\left[\left\|u_{\text {in }}\right\|_{L^{2}\left(\Gamma_{-}\right)}+\|f\|_{L^{2}(X \times \Omega)}\right] .
$$

The above result is summarized in the form of a theorem, as follows.

THEOREM 3.1. Under the assumptions (2.6)-(2.7) and (2.13), the problem (2.11)(2.12) has a unique solution $u \in H^{1,2}(X \times \Omega)$, and this solution is Lipschitz continuous with respect to the source function $f$ and the boundary condition $u_{\mathrm{in}}$ : Given $f_{1}, f_{2} \in$ $L^{2}(X \times \Omega)$ and $u_{\mathrm{in}, 1}, u_{\mathrm{in}, 2} \in L^{2}\left(\Gamma_{-}\right)$, for $k=1,2$, if $u_{k}=u\left(f_{k}, u_{\mathrm{in}, \mathrm{k}}\right) \in H^{1,2}(X \times \Omega)$ is the solution of the problem (2.11)-(2.12) with $f=f_{k}$ and $u_{\mathrm{in}}=u_{\mathrm{in}, \mathrm{k}}$, then for some constant $c$ depending only on $\mu_{t}, \mu_{s}$, and $X$ we have the bound

$$
\left\|u_{1}-u_{2}\right\|_{H^{1,2}(X \times \Omega)} \leq c\left[\left\|u_{\mathrm{in}, 1}-u_{\mathrm{in}, 2}\right\|_{L^{2}\left(\Gamma_{-}\right)}+\left\|f_{1}-f_{2}\right\|_{L^{2}(X \times \Omega)}\right] .
$$

In the study of some XDT problems, it is advantageous to consider MLLE (2.11) in an alternative form,

$$
\boldsymbol{\omega} \cdot \boldsymbol{\nabla} u+\mu_{a} u=\mu_{s}\left(\left(I-\alpha \Delta^{*}\right)^{-1} u-u\right)+f \quad \text { in } X \times \Omega .
$$

Note that the condition (2.6) is equivalent to

$$
\mu_{a}, \mu_{s} \in L^{\infty}(X), \quad \mu_{s} \geq 0 \text { a.e. in } X, \quad \mu_{a} \geq c_{0}>0 \text { a.e. in } X .
$$

At the end of this section, we provide a positivity property for the model (2.11)(2.12). This property is required for the model to be physically meaningful.

Proposition 3.2. Assume (2.6)-(2.7) and (2.13). If $f \geq 0$ a.e. in $X \times \Omega$ and $u_{\text {in }} \geq 0$ a.e. on $\Gamma_{-}$, then for the solution of $(2.11)-(2.12), u \geq 0$ a.e. in $X \times \Omega$.

Proof. From (3.2),

$$
u=(I-A)^{-1} F=\sum_{j=0}^{\infty} A^{j} F .
$$

Copyright $@$ by SIAM. Unauthorized reproduction of this article is prohibited. 
By the assumptions, $F \geq 0$ a.e. in $X \times \Omega$. Let us prove that this implies $A F \geq 0$ a.e. in $X \times \Omega$. By definition of $A$,

$$
A F(\boldsymbol{z}+s \boldsymbol{\omega}, \boldsymbol{\omega})=\lambda(s)^{-1} \int_{s_{-}}^{s} \lambda(t) \mu_{s}(\boldsymbol{z}+t \boldsymbol{\omega}) G(\boldsymbol{z}+t \boldsymbol{\omega}, \boldsymbol{\omega}) d t,
$$

where $G=\left(I-\alpha \Delta^{*}\right)^{-1} F$, and similar to (3.5), for a.e. $\boldsymbol{x} \in X, G(\boldsymbol{x}, \cdot) \in H^{1}(\Omega)$ and

$$
\int_{\Omega}\left(G v+\alpha \nabla^{*} G \cdot \nabla^{*} v\right) d \sigma(\boldsymbol{\omega})=\int_{\Omega} F v d \sigma(\boldsymbol{\omega}) \quad \forall v \in H^{1}(\Omega) .
$$

Take $v=G^{-}$to obtain

$$
\int_{\Omega}\left(\left|G^{-}\right|^{2}+\alpha\left|\nabla^{*} G^{-}\right|^{2}\right) d \sigma(\boldsymbol{\omega})=\int_{\Omega} F G^{-} d \sigma(\boldsymbol{\omega}) \leq 0
$$

Hence, $G^{-}=0$; i.e., $G \geq 0$ a.e. in $X \times \Omega$. Thus, $A F \geq 0$ a.e. in $X \times \Omega$, and then for any positive integer $j, A^{j} F \geq 0$ a.e. in $X \times \Omega$. Therefore, $u \geq 0$ a.e. in $X \times \Omega$.

4. An iteration method and its convergence. We now introduce and study an iteration method for solving the problem defined by (2.15) and (2.12)-(2.14). Let $w^{(0)}$ be an initial guess; e.g., we may take $w^{(0)}=0$. Then, for $n=1,2, \ldots$, define $u^{(n)}$ and $w^{(n)}$ as follows:

$$
\begin{aligned}
\boldsymbol{\omega} \cdot \nabla u^{(n)}+\mu_{t} u^{(n)} & =\mu_{s} w^{(n-1)}+f \quad \text { in } X \times \Omega, \\
u^{(n)} & =u_{\text {in }} \quad \text { on } \Gamma_{-}, \\
w^{(n)} & =\left(I-\alpha \Delta^{*}\right)^{-1} u^{(n)} .
\end{aligned}
$$

Throughout this section, we assume that (2.6)-(2.7) and (2.13) are valid. For a convergence analysis of the iteration method, denote the iteration errors by $e_{u}^{(n)}:=$ $u-u^{(n)}$ and $e_{w}^{(n)}=w-w^{(n)}$. Then we have the error relations

$$
\begin{aligned}
\boldsymbol{\omega} \cdot \boldsymbol{\nabla} e_{u}^{(n)}+\mu_{t} e_{u}^{(n)} & =\mu_{s} e_{w}^{(n-1)} \quad \text { in } X \times \Omega, \\
e_{u}^{(n)} & =0 \quad \text { on } \Gamma_{-}, \\
e_{w}^{(n)} & =\left(I-\alpha \Delta^{*}\right)^{-1} e_{u}^{(n)} .
\end{aligned}
$$

Similar to (3.4) and (3.8), we have

$$
\begin{aligned}
\left\|\mu_{t}^{1 / 2} e_{u}^{(n)}\right\|_{L^{2}(X \times \Omega)} & \leq \kappa\left\|\mu_{t}^{1 / 2} e_{w}^{(n-1)}\right\|_{L^{2}(X \times \Omega)}, \\
\left\|\mu_{t}^{1 / 2} e_{w}^{(n-1)}\right\|_{L^{2}(X \times \Omega)} & \leq\left\|\mu_{t}^{1 / 2} e_{u}^{(n-1)}\right\|_{L^{2}(X \times \Omega)} .
\end{aligned}
$$

Thus,

$$
\left\|\mu_{t}^{1 / 2} e_{u}^{(n)}\right\|_{L^{2}(X \times \Omega)} \leq \kappa\left\|\mu_{t}^{1 / 2} e_{u}^{(n-1)}\right\|_{L^{2}(X \times \Omega)}
$$

and so

$$
\begin{aligned}
\left\|\mu_{t}^{1 / 2} e_{u}^{(n)}\right\|_{L^{2}(X \times \Omega)} & \leq \kappa^{n}\left\|\mu_{t}^{1 / 2} e_{u}^{(0)}\right\|_{L^{2}(X \times \Omega)} \rightarrow 0 \quad \text { as } n \rightarrow \infty, \\
\left\|\mu_{t}^{1 / 2} e_{w}^{(n)}\right\|_{L^{2}(X \times \Omega)} & \leq\left\|\mu_{t}^{1 / 2} e_{u}^{(n)}\right\|_{L^{2}(X \times \Omega)} \rightarrow 0 \quad \text { as } n \rightarrow \infty .
\end{aligned}
$$

Copyright $@$ by SIAM. Unauthorized reproduction of this article is prohibited. 
From (4.4),

$$
\boldsymbol{\omega} \cdot \boldsymbol{\nabla} e_{u}^{(n)}=\mu_{s} e_{w}^{(n-1)}-\mu_{t} e_{u}^{(n)} .
$$

Therefore, we further have the convergence of $u^{(n)} \rightarrow u$ in $H^{1,2}(X \times \Omega)$ :

$$
\left\|e_{u}^{(n)}\right\|_{H^{1,2}(X \times \Omega)} \leq c\left[\left\|e_{w}^{(n-1)}\right\|_{L^{2}(X \times \Omega)}+\left\|e_{u}^{(n)}\right\|_{L^{2}(X \times \Omega)}\right] \rightarrow 0 \quad \text { as } n \rightarrow \infty .
$$

The same convergence statement holds for the iteration method applied to the discretization of the problem defined by (2.15) and (2.12)-(2.14). The iteration method is used in numerical examples of section 7 .

5. XDT problems. In XDT, we try to reconstruct the scattering coefficient $\mu_{s}$. We apply a pencil beam to a part of the boundary $\partial X$ and get angularly resolved or averaged measurements on $\Gamma_{+}$. We do such experiments a few times and determine $\mu_{s}$ by matching the predictions from the MLLE with the measured data on $\Gamma_{+}$. More precisely, let $k_{0}$ be the number of experiments. For $k=1, \ldots, k_{0}$, let $\gamma_{k} \subset \partial X$ be the part of the spatial boundary where the pencil beam passes, let $\boldsymbol{\omega}_{k} \in \Omega$ be the direction of the pencil beam, and define the idealized inflow value function

$$
\tilde{u}_{\mathrm{in}, k}(\boldsymbol{x}, \boldsymbol{\omega})=\chi_{\gamma_{k}}(\boldsymbol{x}) \delta\left(\boldsymbol{\omega}-\boldsymbol{\omega}_{k}\right) .
$$

Here, $\chi_{\gamma_{k}}$ is the characteristic function of the subset $\gamma_{k}$, and $\delta(\cdot)$ is the Dirac delta function. The inflow value function $\tilde{u}_{\text {in }, k}$ of (5.1) is highly idealized in that the function value is infinity in one direction $\boldsymbol{\omega}=\boldsymbol{\omega}_{k}$ and is zero along all other directions. In XDT, we approximate the function $\tilde{u}_{\text {in }, k}$ by

$$
u_{\mathrm{in}, k}(\boldsymbol{x}, \boldsymbol{\omega})=\chi_{\gamma_{k}}(\boldsymbol{x}) \delta_{k}(\boldsymbol{\omega})
$$

where the function $\delta_{k}(\boldsymbol{\omega})$ is an approximation of $\delta\left(\boldsymbol{\omega}-\boldsymbol{\omega}_{k}\right)$; i.e., it is nonnegative, integrates to one, and has a small support around the argument $\boldsymbol{\omega}=\boldsymbol{\omega}_{k}$. As an example, let $B_{k} \subset \Omega$ be a small neighborhood of $\boldsymbol{\omega}_{k}$ on $\Omega$ so that its surface area $\left|B_{k}\right|$ is a small positive number. Then we may take

$$
\delta_{k}(\boldsymbol{\omega})= \begin{cases}\frac{1}{\left|B_{k}\right|}, & \boldsymbol{\omega} \in B_{k}, \\ 0, & \boldsymbol{\omega} \in \Omega \backslash B_{k} .\end{cases}
$$

There are two possible forms for XDT problems, depending on whether the total attenuation coefficient $\mu_{t}$ is known or the absorption coefficient $\mu_{a}$ is known. In the case where $\mu_{t}$ is known, the forward problems for the XDT are

$$
\begin{aligned}
\boldsymbol{\omega} \cdot \boldsymbol{\nabla} u_{k}+\mu_{t} u_{k} & =\mu_{s}\left(I-\alpha \Delta^{*}\right)^{-1} u_{k} \quad \text { in } X \times \Omega, \\
u_{k} & =u_{\mathrm{in}, k} \quad \text { on } \Gamma_{-} .
\end{aligned}
$$

In the case where $\mu_{a}$ is known, we replace (5.3) by (cf. (3.13))

$$
\boldsymbol{\omega} \cdot \boldsymbol{\nabla} u_{k}+\mu_{a} u_{k}=\mu_{s}\left(\left(I-\alpha \Delta^{*}\right)^{-1} u_{k}-u_{k}\right) \quad \text { in } X \times \Omega .
$$

In this paper, we focus our study on the case where $\mu_{t}$ is known. With a slight modification of the arguments, all the discussion based on (5.3) can be extended to that based on (5.5). 
For $1 \leq k \leq k_{0}$, denote the angularly resolved measurement by $u_{\text {meas }, k}$ corresponding to the inflow boundary condition (5.2). Then the XDT problem with angularly resolved measurements is to determine $\mu_{s}(\boldsymbol{x})$ such that for $k=1, \ldots, k_{0}$ the solution $u_{k} \equiv u_{k}\left(\mu_{s}\right)$ of the problem (5.3)-(5.4) satisfies

$$
u_{k}(\boldsymbol{x}, \boldsymbol{\omega})=u_{\text {meas }, k}(\boldsymbol{x}, \boldsymbol{\omega}) \quad \text { for }(\boldsymbol{x}, \boldsymbol{\omega}) \in \Gamma_{+} .
$$

Let $Q_{a d} \subset Q_{0}:=L^{2}(X)$ be an admissible set for the coefficient function $\mu_{s}$. The set $Q_{a d}$ is assumed to be closed and incorporates the constraint $0 \leq \mu_{s} \leq \tilde{\kappa} \mu_{t}$ for some constant $\tilde{\kappa}<1$. Moreover, based on considerations for applications, functions in $Q_{a d}$ are assumed to be piecewise constants or piecewise smooth functions from finite dimensional spaces. Similar to RTE-based inverse problems [8], the XDT problem is ill-posed and is solved with regularization. Introduce the regularized functional

$$
J_{\varepsilon}\left(\mu_{s}\right)=\frac{1}{2} \sum_{k=1}^{k_{0}}\left\|u_{k}-u_{\text {meas }, k}\right\|_{L^{2}\left(\Gamma_{+}\right)}^{2}+\varepsilon R\left(\mu_{s}\right), \quad \varepsilon \geq 0 .
$$

We assume the regularization function $R(\mu)$ satisfies a lower semicontinuity condition:

$$
\mu_{n} \rightarrow \mu \text { in } Q_{0} \text { as } n \rightarrow \infty \quad \Longrightarrow \quad R(\mu) \leq \liminf _{n \rightarrow \infty} R\left(\mu_{n}\right) .
$$

Then the regularized inverse problem is as follows:

Find $\mu_{s} \in Q_{a d}$ such that $J_{\varepsilon}\left(\mu_{s}\right)$ is minimal possible over $Q_{a d}$.

There are a variety of possible choices for the regularization function $R(\mu)$, e.g., $R(\mu)=\frac{1}{2}\|\mu\|_{Q_{0}}^{2}$ or $|\mu|_{T V}$, where $|\mu|_{T V}$ stands for the total variation of $\mu[6,11]$. For $\mathrm{XDT}$, these choices satisfy the condition (5.8). Moreover, we may adopt the Bregman method $[27,16]$, iterative regularization procedures with the use of Bregman distances, to replace the above form of $\left(P_{\varepsilon}\right)$.

We have the following result regarding problem $\left(P_{\varepsilon}\right)$.

THEOREM 5.1. Under the stated assumptions on the set $Q_{a d}$ and the regularization function $R$, for any $\varepsilon \geq 0$, problem $\left(P_{\varepsilon}\right)$ has a solution.

Proof. Denote

$$
j_{0}:=\inf _{\mu \in Q_{a d}} J_{\varepsilon}(\mu)
$$

Let $\left\{\mu_{n}\right\} \subset Q_{a d}$ be a minimizing sequence,

$$
J_{\varepsilon}\left(\mu_{n}\right) \rightarrow j_{0} \quad \text { as } n \rightarrow \infty .
$$

Since $\left\{\mu_{n}\right\}$ is a bounded sequence in a finite dimensional space, we can find a subsequence $\left\{\mu_{n_{j}}\right\} \subset\left\{\mu_{n}\right\}$ and a function $\mu$ such that

$$
\mu_{n_{j}} \rightarrow \mu \quad \text { in } L^{\infty}(X) \text { as } j \rightarrow \infty .
$$

The limit $\mu \in Q_{a d}$ because $Q_{a d}$ is closed.

For $1 \leq k \leq k_{0}$, denote by $u_{k}$ the solution of the problem (5.3)-(5.4) with $\mu_{s}$ replaced by $\mu$ :

$$
\begin{aligned}
\boldsymbol{\omega} \cdot \boldsymbol{\nabla} u_{k}+\mu_{t} u_{k} & =\mu\left(I-\alpha \Delta^{*}\right)^{-1} u_{k} \quad \text { in } X \times \Omega, \\
u_{k} & =u_{\text {in }, k} \quad \text { on } \Gamma_{-} .
\end{aligned}
$$

Copyright $@$ by SIAM. Unauthorized reproduction of this article is prohibited. 
Define $u_{k, n_{j}}$ to be the solution of the problem (5.3)-(5.4) with $\mu_{s}$ replaced by $\mu_{n_{j}}$ :

$$
\begin{aligned}
\boldsymbol{\omega} \cdot \boldsymbol{\nabla} u_{k, n_{j}}+\mu_{t} u_{k, n_{j}} & =\mu_{n_{j}}\left(I-\alpha \Delta^{*}\right)^{-1} u_{k, n_{j}} \quad \text { in } X \times \Omega, \\
u_{k, n_{j}} & =u_{\text {in }, k} \quad \text { on } \Gamma_{-} .
\end{aligned}
$$

Then the difference $e_{k, n_{j}}:=u_{k, n_{j}}-u_{k}$ satisfies

$$
\begin{aligned}
\boldsymbol{\omega} \cdot \nabla e_{k, n_{j}}+\mu_{t} e_{k, n_{j}} & =\mu_{n_{j}}\left(I-\alpha \Delta^{*}\right)^{-1} e_{k, n_{j}}+\left(\mu_{n_{j}}-\mu\right)\left(I-\alpha \Delta^{*}\right)^{-1} u_{k} \quad \text { in } X \times \Omega, \\
e_{k, n_{j}} & =0 \quad \text { on } \Gamma_{-} .
\end{aligned}
$$

Applying Theorem 3.1, we have

$$
\begin{aligned}
\left\|e_{k, n_{j}}\right\|_{H^{1,2}(X \times \Omega)} & \leq c\left\|\left(\mu_{n_{j}}-\mu\right)\left(I-\alpha \Delta^{*}\right)^{-1} u_{k}\right\|_{L^{2}(X \times \Omega)} \\
& \leq c\left\|\mu_{n_{j}}-\mu\right\|_{L^{\infty}(X)}\left\|\left(I-\alpha \Delta^{*}\right)^{-1} u_{k}\right\|_{L^{2}(X \times \Omega)} \\
& \rightarrow 0 \text { as } j \rightarrow \infty .
\end{aligned}
$$

So for $1 \leq k \leq k_{0}$ we have

$$
u_{k, n_{j}} \rightarrow u_{k} \text { in } H^{1,2}(X \times \Omega) \text { as } j \rightarrow \infty .
$$

In particular, this implies, by (2.8),

$$
u_{k, n_{j}} \rightarrow u_{k} \quad \text { in } L^{2}\left(\Gamma_{+}\right) \text {as } j \rightarrow \infty .
$$

Now consider the value $J_{\varepsilon}(\mu)$. We write

$$
\begin{aligned}
J_{\varepsilon}(\mu)= & \frac{1}{2} \sum_{k=1}^{k_{0}}\left\|\left(u_{k, n_{j}}-u_{\text {meas }, k}\right)+\left(u_{k}-u_{k, n_{j}}\right)\right\|_{L^{2}\left(\Gamma_{+}\right)}^{2}+\varepsilon R(\mu) \\
= & J_{\varepsilon}\left(\mu_{n_{j}}\right)+\frac{1}{2} \sum_{k=1}^{k_{0}}\left\|u_{k}-u_{k, n_{j}}\right\|_{L^{2}\left(\Gamma_{+}\right)}^{2} \\
& +\sum_{k=1}^{k_{0}}\left(u_{k, n_{j}}-u_{\text {meas }, k}, u_{k}-u_{k, n_{j}}\right)_{L^{2}\left(\Gamma_{+}\right)}+\varepsilon\left(R(\mu)-R\left(\mu_{n_{j}}\right)\right) .
\end{aligned}
$$

Recall the limiting relations (5.9) and (5.10) as $j \rightarrow \infty$. Letting $j \rightarrow \infty$ in the above equalities, we have

$$
J_{\varepsilon}(\mu) \leq \liminf _{j \rightarrow \infty} J_{\varepsilon}\left(\mu_{n_{j}}\right)=j_{0} .
$$

Therefore, $J_{\varepsilon}(\mu)=j_{0}$, and $\mu$ is a solution of problem $\left(P_{\varepsilon}\right)$.

We turn to consider an XDT problem with angularly averaged measurements. Introduce the measurement operator $M_{k}$ as defined by the formula

$$
M_{k}\left(\mu_{s} ; \boldsymbol{x}\right):=\int_{\boldsymbol{\omega} \in \Omega, \boldsymbol{\omega} \cdot \boldsymbol{\nu}(\boldsymbol{x})>0} u_{k}(\boldsymbol{x}, \boldsymbol{\omega}) \boldsymbol{\omega} \cdot \boldsymbol{\nu}(\boldsymbol{x}) d \sigma(\boldsymbol{\omega}), \quad \boldsymbol{x} \in \partial X,
$$

with $u_{k} \equiv u_{k}\left(\mu_{s} ; \boldsymbol{x}\right)$ the solution of the boundary value problem (5.3)-(5.4). By (2.8), $M_{k}\left(\mu_{s} ; \cdot\right) \in L^{2}(\partial X)$ is well defined. For $1 \leq k \leq k_{0}$, denote by $m_{k}$ the angularly averaged measurement on the spatial boundary $\partial X$. Then the XDT problem with

Copyright $@$ by SIAM. Unauthorized reproduction of this article is prohibited. 
angularly averaged measurements is to determine $\mu_{s}(\boldsymbol{x})$ such that for $k=1, \ldots, k_{0}$ the solution $u_{k} \equiv u_{k}\left(\mu_{s}\right)$ of the problem (5.3)-(5.4) satisfies

$$
M_{k}\left(\mu_{s} ; \boldsymbol{x}\right)=m_{k}(\boldsymbol{x}), \quad \boldsymbol{x} \in \partial X .
$$

The regularized inverse problem is still of the form $\left(P_{\varepsilon}\right)$, where instead of $(5.7)$ the regularized functional is

$$
J_{\varepsilon}\left(\mu_{s}\right)=\frac{1}{2} \sum_{k=1}^{k_{0}}\left\|M_{k}\left(\mu_{s}\right)-m_{k}\right\|_{L^{2}(\partial X)}^{2}+\varepsilon R\left(\mu_{s}\right), \quad \varepsilon \geq 0 .
$$

A slight modification of the proof of Theorem 5.1 shows that the statement of the theorem is valid also for XDT problem with angularly averaged measurements.

6. Discretizations. First, consider the discretization of a general form of the forward problem,

$$
\begin{aligned}
\boldsymbol{\omega} \cdot \boldsymbol{\nabla} u(\boldsymbol{x}, \boldsymbol{\omega})+\mu_{t}(\boldsymbol{x}) u(\boldsymbol{x}, \boldsymbol{\omega}) & =\mu_{s}(\boldsymbol{x}) w(\boldsymbol{x}, \boldsymbol{\omega})+f(\boldsymbol{x}, \boldsymbol{\omega}), \quad(\boldsymbol{x}, \boldsymbol{\omega}) \in X \times \Omega, \\
u(\boldsymbol{x}, \boldsymbol{\omega}) & =u_{\text {in }}(\boldsymbol{x}, \boldsymbol{\omega}), \quad(\boldsymbol{x}, \boldsymbol{\omega}) \in \Gamma_{-}, \\
\left(I-\alpha(\boldsymbol{x}) \Delta^{*}\right) w(\boldsymbol{x}, \boldsymbol{\omega}) & =u(\boldsymbol{x}, \boldsymbol{\omega}), \quad(\boldsymbol{x}, \boldsymbol{\omega}) \in X \times \Omega .
\end{aligned}
$$

A weak formulation of (6.3) is given by (3.5).

Choose a set of nodes $\left\{\boldsymbol{\omega}_{l}\right\}_{l=1}^{L}$ on the unit sphere $\Omega$. Let $W^{h}$ be a finite element space with a nodal basis $\left\{\phi_{l}^{\omega}(\boldsymbol{\omega})\right\}_{l=1}^{L}$ corresponding to the nodes $\left\{\boldsymbol{\omega}_{l}\right\}_{l=1}^{L}$. By a nodal basis we refer to the property $\phi_{l}^{\omega}\left(\boldsymbol{\omega}_{m}\right)=\delta_{l m}$ for $1 \leq l, m \leq L$. Let $\bar{X}=\cup_{K \in \mathcal{T}^{h}} K$ be a finite element partition of $\bar{X}$, and let $U^{h}$ be a corresponding finite element space. Denote by $\left\{\phi_{i}^{x}(\boldsymbol{x})\right\}_{i=1}^{I}$ a basis of $U^{h}$. The numerical solution of the problem (6.1)-(6.3) is expressed as follows:

$$
u^{h}(\boldsymbol{x}, \boldsymbol{\omega})=\sum_{i, l} u_{i l}^{h} \phi_{i}^{x}(\boldsymbol{x}) \phi_{l}^{\omega}(\boldsymbol{\omega}), \quad w^{h}(\boldsymbol{x}, \boldsymbol{\omega})=\sum_{i, l} w_{i l}^{h} \phi_{i}^{x}(\boldsymbol{x}) \phi_{l}^{\omega}(\boldsymbol{\omega})
$$

where $\sum_{i, l}$ stands for $\sum_{i=1}^{I} \sum_{l=1}^{L}$. For convenience, we will use the following notation:

$$
\begin{aligned}
u_{l}^{h}(\boldsymbol{x})=\sum_{i} u_{i l}^{h} \phi_{i}^{x}(\boldsymbol{x}), & u_{i}^{h}(\boldsymbol{\omega})=\sum_{l} u_{i l}^{h} \phi_{l}^{\omega}(\boldsymbol{\omega}), \\
w_{l}^{h}(\boldsymbol{x})=\sum_{i} w_{i l}^{h} \phi_{i}^{x}(\boldsymbol{x}), & w_{i}^{h}(\boldsymbol{\omega})=\sum_{l} w_{i l}^{h} \phi_{l}^{\omega}(\boldsymbol{\omega}) .
\end{aligned}
$$

We follow [17] to develop a discrete-ordinate discontinuous Galerkin method for (6.1) and the boundary condition (6.2), and use the regular finite element method for (3.5). The resulting numerical method for solving the problem (6.1)-(6.3) is the following. For $1 \leq l \leq L$,

$$
\begin{array}{r}
\int_{\partial K} \widehat{u_{l}^{h}} \boldsymbol{\omega}_{l} \cdot \boldsymbol{\nu}_{K} \phi_{j}^{x} d \sigma(\boldsymbol{x})-\int_{K} u_{l}^{h} \boldsymbol{\omega}_{l} \cdot \boldsymbol{\nabla} \phi_{j}^{x} d x+\int_{K} \mu_{t} u_{l}^{h} \phi_{j}^{x} d x \\
=\int_{K} \mu_{s} w_{l}^{h} \phi_{j}^{x} d x+\int_{K} f_{l} \phi_{j}^{x} d x, \quad 1 \leq j \leq L, \forall K \in \mathcal{T}^{h},
\end{array}
$$

and for $1 \leq i \leq I$,

$$
\int_{\Omega}\left(w_{i}^{h} \phi_{m}^{\omega}+\alpha \nabla^{*} w_{i}^{h} \cdot \nabla^{*} \phi_{m}\right) d \sigma(\boldsymbol{\omega})=\int_{\Omega} u_{i}^{h} \phi_{m}^{\omega} d \sigma(\boldsymbol{\omega}), \quad 1 \leq m \leq L .
$$

Copyright (c) by SIAM. Unauthorized reproduction of this article is prohibited. 
Here, $f_{l}(\boldsymbol{x}):=f\left(\boldsymbol{x}, \boldsymbol{\omega}_{l}\right), \boldsymbol{\nu}_{K}$ is the outward unit normal on $\partial K, \widehat{u_{l}^{h}}$ is the so-called numerical flux, and we may take

$$
\widehat{u_{l}^{h}}(\boldsymbol{x})= \begin{cases}u_{\mathrm{in}}^{h}(\boldsymbol{x}) & \text { if }\left(\boldsymbol{x}, \boldsymbol{\omega}_{l}\right) \in \Gamma_{-}, \\ \lim _{\varepsilon \rightarrow 0+} u_{l}^{h}\left(\boldsymbol{x}-\varepsilon \boldsymbol{\omega}_{l}\right) & \text { otherwise, }\end{cases}
$$

where $u_{\mathrm{in}}^{h}(\boldsymbol{x})$ is an approximation of $u_{\mathrm{in}}(\boldsymbol{x})$ from the finite element space $U^{h}$.

For a numerical approximation of XDT problems, we denote the discrete admissible set $Q_{a d}^{h_{x}}:=Q_{a d} \cap Q_{0}^{h_{x}}$, where $Q_{0}^{h_{x}}$ is a finite element space approximating $Q_{0}$, e.g., using piecewise constants. Then the numerical scheme for problem $\left(P_{\varepsilon}\right)$ is

$\left(P_{\varepsilon}^{h}\right) \quad$ Find $\mu_{s}^{h} \in Q_{a d}^{h_{x}}$ such that $J_{\varepsilon}^{h}\left(\mu_{s}^{h}\right)$ is minimal possible over $Q_{a d}^{h_{x}}$.

Here,

$$
J_{\varepsilon}^{h}\left(\mu_{s}^{h}\right)=\frac{1}{2} \sum_{k=1}^{k_{0}}\left\|u_{k}^{h}-u_{\text {meas }, k}\right\|_{L^{2}\left(\Gamma_{+}\right)}^{2}+\varepsilon R\left(\mu_{s}^{h}\right)
$$

for the case of angularly resolved measurement;

$$
J_{\varepsilon}^{h}\left(\mu_{s}^{h}\right)=\frac{1}{2} \sum_{k=1}^{k_{0}}\left\|M_{k}^{h}\left(\mu_{s}^{h}\right)-m_{k}\right\|_{L^{2}(\partial X)}^{2}+\varepsilon R\left(\mu_{s}^{h}\right)
$$

for the case of angularly averaged measurement; $u_{k}^{h}$ is the numerical solution defined above for the problem (6.1)-(6.3) with $f=0, u$ replaced by $u_{k}, w$ replaced by $w_{k}$, and $u_{\text {in }}$ replaced by $u_{\text {in, } k}$; and

$$
M_{k}^{h}\left(\mu_{s}^{h} ; \boldsymbol{x}\right):=\int_{\boldsymbol{\omega} \in \Omega, \boldsymbol{\omega} \cdot \boldsymbol{\nu}(\boldsymbol{x})>0} u_{k}^{h}(\boldsymbol{x}, \boldsymbol{\omega}) \boldsymbol{\omega} \cdot \boldsymbol{\nu}(\boldsymbol{x}) d \sigma(\boldsymbol{\omega}), \quad \boldsymbol{x} \in \partial X .
$$

Similar to Theorem 5.1, the discrete problem $\left(P_{\varepsilon}^{h}\right)$ has a solution for any $\varepsilon \geq 0$. Convergence and error estimation for the numerical method (6.5)-(6.6) in solving the forward problem and for the numerical scheme in solving XDT problem are subjects for further studies.

7. Numerical examples. We present here some preliminary numerical examples on solving MLLE and XDT problems with angularly resolved measurement. The purpose is to show that the mathematical framework developed in this paper for XDT is suitable for reconstruction of the scattering coefficient. Substantial further effort is required to explore efficient and effective algorithms to simulate XDT problems.

First we consider solving a problem of the type (6.3):

$$
w(\boldsymbol{\omega})-\alpha \Delta^{*} w(\boldsymbol{\omega})=f(\boldsymbol{\omega}), \quad \boldsymbol{\omega} \in \Omega,
$$

where $\alpha \geq 0$ and $f \in L^{2}(\Omega)$ are given. The corresponding weak formulation is

$$
w \in H^{1}(\Omega): \quad \int_{\Omega}\left(w v+\alpha \boldsymbol{\nabla}^{*} w \cdot \nabla^{*} v\right) d \sigma(\boldsymbol{\omega})=\int_{\Omega} f v d \sigma(\boldsymbol{\omega}) \quad \forall v \in H^{1}(\Omega) .
$$

We use the standard spherical coordinates, $x(\psi, \theta)=\cos \psi \sin \theta, y(\psi, \theta)=\sin \psi \sin \theta$, $z(\psi, \theta)=\cos \theta$ for $0 \leq \psi<2 \pi, 0 \leq \theta \leq \pi$, and follow [3] to formulate a Galerkin method where the basis functions are piecewise linear on triangular elements of the mesh and bilinear on rectangular elements of the mesh. The partition of the closed domain $G=[0,2 \pi] \times[0, \pi]$ for the variables $\psi$ and $\theta$ in [3] involves two parameters: $n_{\psi}$ 
TABLE 7.1

$L^{2}(\Omega)$ error for Example 7.1.

\begin{tabular}{|l|l|}
\hline$n_{\theta}$ & $e$ \\
\hline 4 & 1.465321 \\
8 & 0.465694 \\
16 & 0.143430 \\
32 & 0.041513 \\
64 & 0.010879 \\
128 & 0.002756 \\
\hline
\end{tabular}

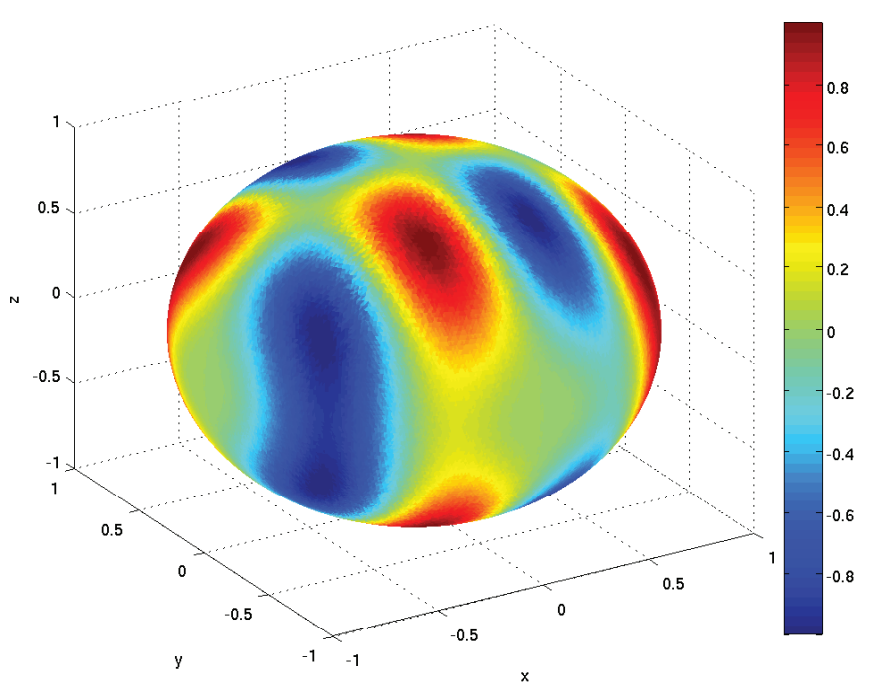

FIG. 7.1. Example 7.1: Numerical solution with $n_{\theta}=128$.

and $n_{\theta}$, where $n_{\psi}$ is usually taken to be 4,6 , or 8 and $n_{\theta}$ is even. Initially, the domain $G$ is divided $n_{\psi}$ times in the $\psi$-direction and $n_{\theta}$ times in the $\theta$-direction. The generated rectangles at $\theta=0, \pi$ remain unchanged, corresponding to the triangles on the unit sphere at the poles. Define $h_{\theta}=\pi / n_{\theta}$. An edge with $\theta=k h_{\theta} \leq \pi / 2,1 \leq k \leq n_{\theta} / 2$, is equally split into $(k-1)$ parts. Then the generated nodes are connected properly, and the generated mesh is reflected with respect to the line $\theta=\pi / 2$. Figure 3 of [3] shows representative partitions of $G$ and the corresponding isotropic triangulation of the unit sphere $\Omega$. We refer the reader to [3] for details of this method, and give one brief example to demonstrate the correctness of implementation.

Example 7.1. Set $\alpha=1$, and choose the function $f$ so that the solution $w$ of the problem $(7.1)$ is $w(\psi, \theta)=\sin (2 \pi x(\psi, \theta)) \sin (\pi y(\psi, \theta))$. In Table 7.1, we report the $L^{2}(\Omega)$ error

$$
e:=\left(\int_{\Omega}\left|w-w^{h}\right|^{2} d \sigma(\boldsymbol{\omega})\right)^{\frac{1}{2}}
$$

for $n_{\psi}=8$ and several values of $n_{\theta}$. We observe a numerical convergence order of 2 . The numerical solution with $n_{\theta}=128$, which is visually indistinguishable from that of the true solution, is depicted in Figure 7.1.

The second example concerns solving the MLLE.

Example 7.2. This is an example of using (6.5)-(6.6) to solve the problem (6.1)(6.3). The spatial domain is $X=(0,1)^{3}$. Let $\alpha=1, \mu_{t}=2$, and $\mu_{s}=1$. We choose 
TABle 7.2

Example 7.2: $L^{2}$ error for several values of $h$ and $n_{\theta}$ with $n_{\psi}=6$.

\begin{tabular}{|l|l|l|l|l|l|l|l|l|}
\hline$h$ & $n_{\theta}=4$ & Order & $n_{\theta}=8$ & Order & $n_{\theta}=12$ & Order & $n_{\theta}=16$ & Order \\
\hline$\frac{\sqrt{2}}{2}$ & 0.3884 & & 0.3461 & & 0.3432 & & 0.3413 & \\
$\frac{\sqrt{2}}{4}$ & 0.1174 & 1.7257 & 0.1003 & 1.7866 & 0.0990 & 1.7936 & 0.0983 & 1.7954 \\
$\frac{\sqrt{2}}{8}$ & 0.0509 & 1.2063 & 0.0300 & 1.7427 & 0.0275 & 1.8496 & 0.0270 & 1.8666 \\
\hline
\end{tabular}

the functions $f$ and $u_{\text {in }}$ so that the true solution to (6.1)-(6.3) is

$$
\begin{aligned}
& u(\boldsymbol{x}, \boldsymbol{\omega}) \\
& \quad=-\frac{3}{2} \cos ^{2} \psi\left\{\cos ^{2} \psi[1+7 \cos (2 \theta)]+8 \sin ^{2} \psi\right\} \sin ^{2} \theta \sin \left(\pi x_{1}\right) \sin \left(\pi x_{2}\right) \sin \left(\pi x_{3}\right) .
\end{aligned}
$$

For a positive integer $n$, we partition $\bar{X}$ into $n^{3}$ subcubes $\left\{X_{i}\right\}$, each with edge length $1 / n$. Denote by $S$ the set of all the centers and vertices of the subcubes. A mesh is generated by creating the Delaunay tesselation of the points in $S$. This is accomplished using the delaunay 3 algorithm in MATLAB. The option "QJ" is passed to delaunay 3 to ensure that no hanging nodes are created. Denote by $h$ the maximum length of the edges of the tetrahedron in the mesh; $h=\sqrt{2} / n$. The local polynomial degree $k=1$.

Table 7.2 gives the error

$$
e:=\left\{\frac{4 \pi}{L} \sum_{l}\left\|u_{h}^{l}-u\left(\cdot, \boldsymbol{\omega}_{l}\right)\right\|_{L^{2}(X)}^{2}\right\}^{\frac{1}{2}}
$$

for $n_{\psi}=6$ and several values of $h$ and $n_{\theta}$. The column "Order" is for the quantity $\operatorname{order}_{i}=\left(\log e_{i}-\log e_{i-1}\right) /\left(\log h_{i}-\log h_{i-1}\right)$, where $e_{i}$ is the error defined above corresponding to the mesh-size $h_{i}$. For small $n_{\theta}$ (e.g., $n_{\theta}=4$ ), notice a deterioration in the convergence order as $h$ decreases from $\sqrt{2} / 4$ to $\sqrt{2} / 8$. The deterioration phenomenon disappears for the chosen values of $h$ when the value of $n_{\theta}$ is increased. For very small values of $h$, to achieve a numerical convergence order around the expected value 2 , both $n_{\theta}$ and $n_{\psi}$ need to be large enough.

For reference, for $n_{\psi}=6$, with $n_{\theta}=4$ there are 18 directional nodes, with $n_{\theta}=8$ there are 98 , with $n_{\theta}=12$ there are 218 , and with $n_{\theta}=16$ there are 386 .

Next, we present some examples on XDT. In these examples, we use the HenyeyGreenstein phase function (2.3) with $g=0.9$.

Example 7.3. Consider the XDT problem of recovering $\mu_{s}$, where $u_{\text {meas }, k}$ is an approximate solution to the MLLE with the following parameters:

$$
\begin{aligned}
& \mu_{s}=\left\{\begin{array}{ll}
1.1 & \text { if } \boldsymbol{x} \in R_{1}, \\
1.4 & \text { otherwise, }
\end{array} \quad \mu_{t}=1.5,\right. \\
& \left.u_{\text {meas }, k}(\boldsymbol{x}, \boldsymbol{\omega})\right|_{\Gamma_{-}}= \begin{cases}\frac{L}{4 \pi} & \text { if } \boldsymbol{x} \in S_{k} \text { and } \boldsymbol{\omega} \text { is around } \boldsymbol{\omega}_{k}, \quad k=1,2 . \\
0 & \text { otherwise },\end{cases}
\end{aligned}
$$

The domain $X=(0,1)^{3}$. The domains $R_{1}$ and $R_{2}:=X \backslash R_{1}$ are illustrated in Figure 7.2. The surfaces $S_{1}$ and $S_{2}$ are illustrated in Figure 7.3. The spatial mesh used to generate $u_{\text {meas }, k}$ is shown in each of the preceding figures and has 96 elements. 


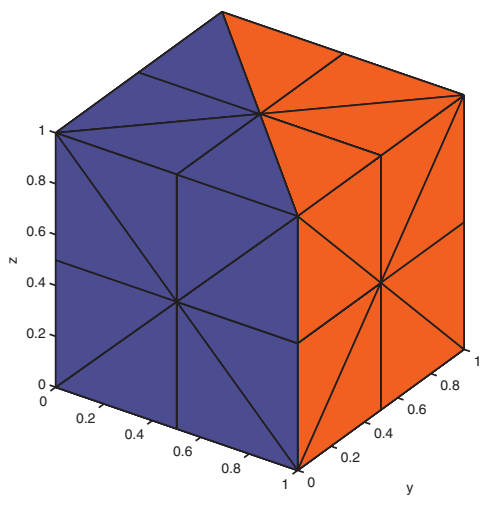

FIG. 7.2. Domains $R_{1}$ and $R_{2}$ in different colors.

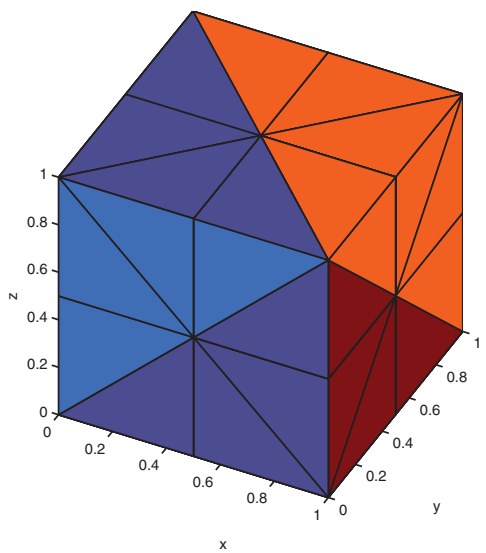

FIG. 7.3. Domains $R_{1}$ and $R_{2}$ with surfaces $S_{1}$ and $S_{2}$ highlighted.

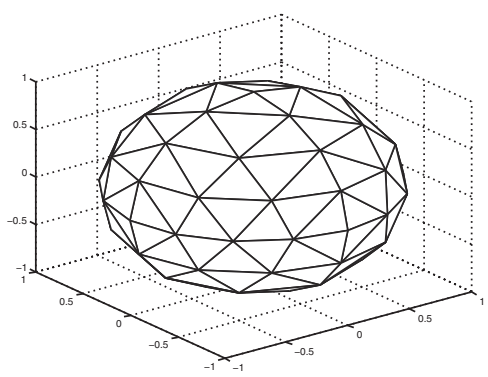

FIG. 7.4. Angular mesh with 66 nodes.

The angular mesh is shown in Figure 7.4 and has 66 nodes. In the above definition $L=66$. Directions $\boldsymbol{\omega}_{1}$ and $\boldsymbol{\omega}_{2}$ are node points of the angular mesh that point from the centers of $S_{1}$ and $S_{2}$, respectively, to the center of the cube $X$.

In order to reconstruct $\mu_{s}$ we use approximate solutions to the MLLE constructed on a spatial mesh with 12 elements. Define

$$
Q_{a d}=\left\{\mu|\mu|_{R_{k}}=\mu_{k} \in \mathbb{R}, 0 \leq \mu_{k} \leq \mu_{t}, k=1,2\right\} .
$$




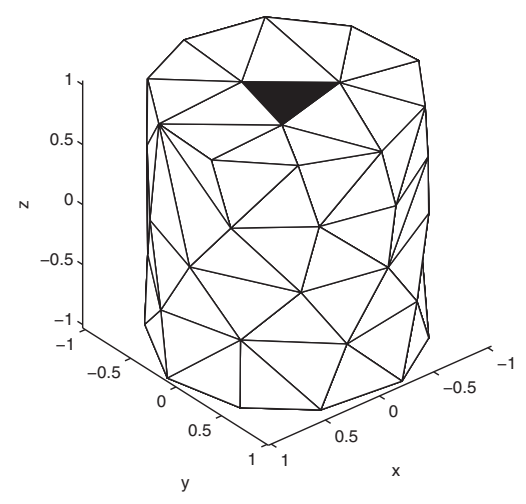

Fig. 7.5. The domains $R_{1}$ and $R_{2}$ in different colors.

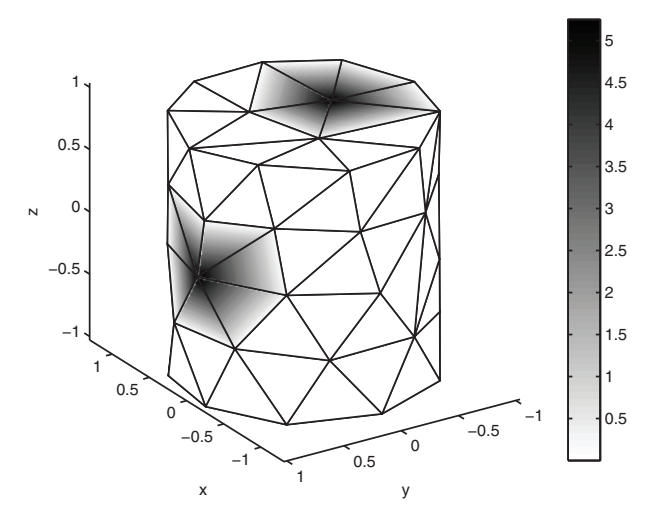

FIG. 7.6. The functions $B_{1}$ and $B_{2}$, with $\boldsymbol{\omega}$ fixed at $\boldsymbol{\omega}_{1}$ and $\boldsymbol{\omega}_{2}$, respectively.

Since two sets of measurements are used to determine two constants that define $\mu_{s}$, we may take $\varepsilon=0$. Denote the reconstruction of $\mu_{s}$ by $\mu_{s}^{r}$. In this case we have

$$
\mu_{s}^{r}(\boldsymbol{x})= \begin{cases}1.0218 & \text { if } \boldsymbol{x} \in R_{1} \\ 1.3483 & \text { otherwise }\end{cases}
$$

which compares well with the true values.

To increase the accuracy of the reconstructed values of $\mu_{s}$, we then solve the problem with $u_{\text {meas, } k}$ computed on a mesh with 768 elements and the approximate solution constructed on a mesh with 96 elements. The result is

$$
\mu_{s}^{r}(\boldsymbol{x})= \begin{cases}1.1008 & \text { if } \boldsymbol{x} \in R_{1} \\ 1.4326 & \text { otherwise }\end{cases}
$$

We observe an improvement in the reconstruction accuracy as the approximate solution becomes more accurate, as expected.

Example 7.4. In this example the domain $X$ is the approximate cylinder shown in Figure 7.5. The regions $R_{1}$ and $R_{2}$ are seen in the same figure. The boundary regions $S_{1}$ and $S_{2}$ are shown in Figure 7.6. The functions $u_{\text {meas, } k}$ are computed from 
approximate solutions of (6.5)-(6.6) corresponding to the data

$$
\mu_{s}=\left\{\begin{array}{ll}
1.1 & \text { if } \boldsymbol{x} \in R_{1}, \\
1.4 & \text { otherwise },
\end{array} \quad \mu_{t}=1.5,\left.\quad u_{\mathrm{in}, k}(\boldsymbol{x}, \boldsymbol{\omega})\right|_{\Gamma_{-}}=B_{k}, \quad k=1,2 .\right.
$$

The boundary value $B_{k}$ is a piecewise linear function whose spatial support is $S_{k}$ and achieves the value $\frac{L}{4 \pi}$ at the center node of $S_{k}$. The directions $\boldsymbol{\omega}_{k}$ are nodes of the angular mesh that point approximately from the center of $S_{k}$ to the center of $X$.

When the mesh for (6.5)-(6.6) in determining $u_{\text {meas }, k}$ has 1608 elements and the mesh for computing $u_{n}$ has 201 elements, we have

$$
\mu_{s}^{r}(\boldsymbol{x})= \begin{cases}1.2267 & \text { if } \boldsymbol{x} \in R_{1} \\ 1.1931 & \text { otherwise }\end{cases}
$$

When the meshes are refined to having 12864 and 1608 elements, respectively,

$$
\mu_{s}^{r}(\boldsymbol{x})= \begin{cases}1.1332 & \text { if } \boldsymbol{x} \in R_{1} \\ 1.3662 & \text { otherwise }\end{cases}
$$

We again see improvement as the approximations of $u_{k}$ become more accurate.

Example 7.5. The geometric setting is the same as in Example 7.3. However, in this example, we assume that values of $\mu_{a}$, rather than of $\mu_{t}$, are known. The functions $u_{\text {meas, } k}$ are calculated on a spatial mesh with 268 elements and an angular mesh with 66 nodes, and are the approximate solutions to the MLLE corresponding to the data

$$
\begin{aligned}
& \mu_{s}=\left\{\begin{array}{ll}
1.1 & \text { if } \boldsymbol{x} \in R_{1}, \\
1.4 & \text { otherwise, }
\end{array} \quad \mu_{a}= \begin{cases}0.4 & \text { if } \boldsymbol{x} \in R_{1}, \\
0.1 & \text { otherwise },\end{cases} \right. \\
& \left.u_{\text {meas }, k}(\boldsymbol{x}, \boldsymbol{\omega})\right|_{\Gamma_{-}}= \begin{cases}\frac{L}{4 \pi} & \text { if } \boldsymbol{x} \in S_{k} \text { and } \boldsymbol{\omega} \text { is around } \boldsymbol{\omega}_{k}, \quad k=1,2 . \\
0 & \text { otherwise }\end{cases}
\end{aligned}
$$

The approximate solutions for $u_{k}$ are calculated on a spatial mesh with 96 elements and an angular mesh with 66 nodes. In this case we get

$$
\mu_{s}^{r}(\boldsymbol{x})= \begin{cases}1.0965 & \text { if } \boldsymbol{x} \in R_{1} \\ 1.3680 & \text { otherwise }\end{cases}
$$

We see a good agreement between the reconstructed values and true values of $\mu_{s}$.

8. Concluding remarks. In this paper, we provided a preliminary theoretical study of x-ray dark-field tomography (XDT) for the reconstruction of the scattering parameter in a strongly forward-peaked biological medium. We showed the wellposedness of the forward problem in XDT and studied XDT problems with angularly resolved measurements or angularly averaged measurements. We then introduced numerical methods to solve XDT problems. Numerical examples illustrated the reliability of the numerical results using the proposed methods.

There are many issues related to XDT and are worth effort for further investigations. We list a few topics in the following.

Uniqueness results for XDT. While it is reasonable to expect uniqueness when the number of measurements is larger than or equal to the number of unknowns 
needed to define the scattering coefficient, for practical reconstruction of the scattering parameter it is desirable to use the least number of measurements that guarantees uniqueness. Theoretical results on uniqueness are helpful.

Convergence and error analysis for the numerical methods. Such a convergence analysis and error estimation are important for understanding the performance of the numerical methods in solving MLLE and XDT problems.

Theoretical and numerical comparisons for different choices of the regularization function $R(\mu)$. In the preliminary numerical examples presented in section 7 , the number of measurements is equal to the number of unknowns needed to determine the scattering parameter, and hence no regularization is used. In more realistic simulations, the number of measurements is much smaller than the number of unknowns for the scattering parameter, and regularization plays a fundamental role in dealing with the ill-conditioning of the problem and in controlling data noise. While it is believed that the Bregman-type methods $[27,16]$ are prominent choices for the type of inverse problems we solve in XDT, detailed theoretical analysis and simulation experiments are needed to identify the best choice of the regularization function for XDT.

Acknowledgment. We thank the two anonymous referees for their valuable comments on the first version of this paper.

\section{REFERENCES}

[1] A. Agoshkov, Boundary Value Problems for Transport Equations, Birkhäuser/Cambridge, Boston, MA, 1998.

[2] N. G. Anderson, A. P. Butler, N. J. Scott, N. J. Cook, J. S. Butzer, N. Schleich, M. Firsching, R. Grasset, N. De Ruiter, M. Campbell, and P. H. Butler, Spectroscopic (multi-energy) CT distinguishes iodine and barium contrast material in MICE, European Radiol., 20 (2010), pp. 2126-2134.

[3] T. Apel AND C. Pester, Clement-type interpolation on spherical domains-Interpolation error estimates and application to a posteriori error estimation, IMA J. Numer. Anal., 25 (2005), pp. 310-336.

[4] S. R. ARRIDGe, Optical tomography in medical imaging, Inverse Problems, 15 (1999), pp. R41R93.

[5] K. Atkinson and W. Han, Theoretical Numerical Analysis: A Functional Analysis Framework, 3rd ed., Springer-Verlag, New York, Berlin, 2009.

[6] H. Атtouch, G. Buttazzo, and G. Michaille, Variational Analysis in Sobolev and BV Spaces: Applications to PDEs and Optimization, MPS/SIAM Ser. Optim. 6, Philadelphia, 2006.

[7] G. Avtandilov, A. Dembo, O. Komardin, P. Lazarev, M. Paukshto, L. Shkolnik, and O. ZayratiYants, Human tissue analysis by small-angle X-ray scattering, J. Appl. Crystallog., 33 (2000), pp. 511-514.

[8] G. BAL, Inverse transport theory and applications, Inverse Problems, 25 (2009), 053001.

[9] A. P. H. Butler, N. G. Anderson, R. Tipples, N. Cook, R. Watts, J. Meyer, A. J. Bell, T. R. Melzer, And P. H. Butler, Bio-medical X-ray imaging with spectroscopic pixel detectors, Nuclear Instruments Methods in Phys. Res. A, 591 (2008), pp. 141-146.

[10] L. C. Evans, Partial Differential Equations, American Mathematical Society, Providence, RI, 1998.

[11] L. C. Evans and R. F. Gariepy, Measure Theory and Fine Properties of Functions, CRC Press, Boca Raton, FL, 1992.

[12] M. Fernández, J. Keyriläinen, R. Serimaa, M. Torkkeli, M.-L. Karjalainen-Lindsberg, M. Leidenius, K. von Smitten, M. Tenhunen, S. Fiedler, A. Bravin, T. M. Weiss, and P. SuORTTI, Human breast cancer in vitro: Matching histo-pathology with small-angle $x$-ray scattering and diffraction enhanced x-ray imaging, Phys. Med. Biol., 50 (2005), pp. 29913006.

[13] D. Gilbarg and N. S. Trudinger, Elliptic Partial Differential Equations of Second Order, Springer-Verlag, New York, Berlin, 2001.

Copyright (c) by SIAM. Unauthorized reproduction of this article is prohibited. 
[14] S. J. Glick, Breast CT, Annu. Rev. Biomed. Engrg., 9 (2007), pp. 501-526.

[15] S. J. Glick, S. Thacker, X. Gong, And B. Liu, Evaluating the impact of $x$-ray spectral shape on image quality in flat-panel CT breast imaging, Med. Phys., 34 (2007), pp. 5-24.

[16] T. Goldstein And S. OsheR, The split Bregman method for L1-regularized problems, SIAM J. Imaging Sci., 2 (2009), pp. 323-343.

[17] W. Han, J. Huang, And J. A. EichHolz, Discrete-ordinate discontinuous Galerkin methods for solving the radiative transfer equation, SIAM J. Sci. Comput., 32 (2010), pp. 477-497.

[18] L. Henyey and J. Greenstein, Diffuse radiation in the galaxy, Astrophys. J., 93 (1941), pp. $70-83$.

[19] A. D. Kim And J. B. Keller, Light propagation in biological tissue, J. Opt. Soc. Amer. A, 20 (2003), pp. 92-98.

[20] C. L. LEAKEAS AND E. W. LARSEn, Generalized Fokker-Planck approximations of particle transport with highly forward-peaked scattering, Nuclear Sci. Eng., 137 (2001), pp. 236250.

[21] E. E. Lewis And W. F. Miller, Computational Methods of Neutron Transport, John Wiley \& Sons, New York, 1984.

[22] X. Llopart, M. Campbell, R. Dinapoli, D. San Segundo, and E. Pernigotti, Medipix2: A 64- $k$ pixel readout chip with $55-\mu m$ square elements working in single photon counting mode, IEEE Trans. Nuclear Sci., 49 (2002), pp. 2279-2283.

[23] A. Momose, T. Takeda, Y. Itai, and K. Hirano, Phase-contrast X-ray computed tomography for observing biological soft tissues, Nature Medicine, 2 (1996), pp. 473-475.

[24] C. MÜller, Analysis of Spherical Symmetries in Euclidean Spaces, Springer, New York, 1998.

[25] M. Muttarak, S. Pojchamarnwiputh, and B. Chaiwun, Breast cancer in women under 40 years: Preoperative detection by mammography, Ann. Acad. Med. Singapore, 32 (2003), pp. 433-437.

[26] F. Natterer and F. Wübbeling, Mathematical Methods in Image Reconstruction, Monogr. Math. Model. Comput. 5, SIAM, Philadelphia, 2001.

[27] S. Osher, M. Burger, D. Goldfarb, J. Xu, And W. Yin, An iterative regularization method for total variation-based image restoration, Multiscale Model. Simul., 4 (2005), pp. 460489.

[28] F. Pfeiffer, M. Bech, O. Bunk, P. Kraft, E. F. Eikenberry, Ch. Brönnimann, C. GrünZweig, AND C. DAVID, Hard-X-ray dark-field imaging using a grating interferometer, Nature Materials, 7 (2008), pp. 134-137.

[29] E. D. Pisano, C. Gatsonis E. Hendrick, M. Yaffe, J. K. Baum, S. Acharyya, E. F. Conant, L. L. Fajardo, L. Bassett, C. D’Orsi, R. Jong, and M. Rebner, Diagnostic performance of digital versus film mammography for breast-cancer screening, New England J. Med., 353 (2005), pp. 1773-1783.

[30] G. Wang, W. X. Cong, H. O. Shen, And Y. Zou, Varying collimation for dark-field extraction, Internat. J. Biomed. Imaging, 2009 (2009), 847537.

[31] E. Zeidler, Nonlinear Functional Analysis and Its Applications. I: Fixed-point Theorems, Springer-Verlag, New York, Berlin, 1985.

Copyright (c) by SIAM. Unauthorized reproduction of this article is prohibited. 